PROCEEDINGS OF THE

AMERICAN MATHEMATICAL SOCIETY

Volume 139, Number 6, June 2011, Pages 2073-2085

S 0002-9939(2010)10610-X

Article electronically published on November 3, 2010

\title{
BIFURCATIONS OF MULTIPLE RELAXATION OSCILLATIONS IN POLYNOMIAL LIÉNARD EQUATIONS
}

\author{
P. DE MAESSCHALCK AND F. DUMORTIER
}

(Communicated by Yingfei Yi)

\begin{abstract}
In this paper, we prove the presence of limit cycles of given multiplicity, together with a complete unfolding, in families of (singularly perturbed) polynomial Liénard equations. The obtained limit cycles are relaxation oscillations. Both classical Liénard equations and generalized Liénard equations are treated.
\end{abstract}

\section{INTRODUCTION}

This paper exclusively deals with relaxation oscillations in polynomial Liénard equations. Liénard equations are related to second order scalar differential equations

$$
m x^{\prime \prime}=-g(x, \lambda)-f(x, \lambda) x^{\prime},
$$

where $\lambda \in \Lambda$ is a multi-dimensional parameter in a subset $\Lambda$ of some euclidean space. In the phase plane it can be written as

$$
\left\{\begin{aligned}
m x^{\prime} & =Y, \\
Y^{\prime} & =-g(x, \lambda)-\frac{Y}{m} f(x, \lambda) .
\end{aligned}\right.
$$

By defining $F(x, \lambda)=\int_{0}^{x} f(s, \lambda) d s$ and introducing the variable $y=Y+F(x, \lambda)$, we get an expression in the so-called Liénard plane

$$
\left\{\begin{aligned}
m x^{\prime} & =y-F(x, \lambda), \\
y^{\prime} & =-g(x, \lambda) .
\end{aligned}\right.
$$

Taking $m=\varepsilon \sim 0, \varepsilon>0$, in (11) and introducing a "fast time" by multiplying the former time by $1 / \varepsilon$, we get a singular perturbation problem

$$
\left\{\begin{array}{l}
\dot{x}=y-F(x, \lambda) \\
\dot{y}=-\varepsilon g(x, \lambda)
\end{array}\right.
$$

As observed before, we can also represent (2) in the phase plane by

$$
\left\{\begin{array}{l}
\dot{x}=y \\
\dot{y}=-\varepsilon g(x, \lambda)-y f(x, \lambda) .
\end{array}\right.
$$

Received by the editors March 24, 2010 and, in revised form, May 31, 2010

2010 Mathematics Subject Classification. Primary 37G15, 34E17; Secondary 34C07, 34C26.

Key words and phrases. Slow-fast system, singular perturbations, limit cycles, relaxation oscillation, polynomial Liénard equations, elementary catastrophy.

The first author was supported by the Research Foundation Flanders.

(C)2010 American Mathematical Society Reverts to public domain 28 years from publication 

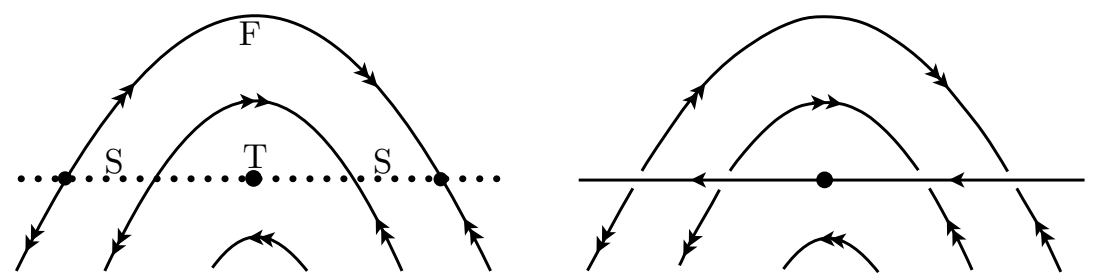

Figure 1. (a) The dynamics of the layer equation and an FSTScycle. (b) The slow dynamics along the slow curve, combined with the fast dynamics

We can work with either (2) or (3) in looking for limit cycles. We are specifically interested in relaxation oscillations, i.e. limit cycles that are subject to two different speeds, one of which is $O(1)$ while the other is $O(\varepsilon)$, for $\varepsilon \rightarrow 0$ (keeping $\varepsilon>0$ ). We limit ourselves to relaxation oscillations of size $O(1)$.

To detect such relaxation oscillations one considers $\varepsilon=0$ in either (2) or (3) and studies the so-called layer equation. These systems have a curve of zeros, called the slow curve. Movements of (2) or (3), with $\varepsilon>0$ small, that are close to that curve of zeros will be "slow" (i.e. move with speed of the order $O(\varepsilon)$ ), while movements close to regular points of the layer equation will be fast (of order $O(1)$ ).

Relaxation oscillations for small $\varepsilon>0$ can only be found near degenerate limit periodic sets of the layer equation, i.e. closed curves combining fast orbits and parts of the slow curve; we call such limit periodic sets slow-fast cycles.

If we only stay near parts of the slow curve where the layer equation is always hyperbolically attracting (or always hyperbolically repelling), then we call such limit periodic sets "common" (and we use the same terminology for the nearby relaxation oscillations for $\varepsilon>0$ ). The common relaxation oscillations that have been studied so far are, as far as we know, all hyperbolic (resp. attracting or repelling depending on the case).

Relaxation oscillations of higher multiplicity, often accompanied by a full unfolding, are known to be possible if the slow-fast cycle contains both hyperbolically attracting and repelling slow curves. Such limit periodic sets, as well as the nearby relaxation oscillations, are said to be "of canard type" or are for short called "canards".

The simplest such canards contain one fast orbit and one slow curve along which the layer equation changes from hyperbolically attracting to hyperbolically repelling at a unique (so-called) "turning point" (see Figure 1(a)).

In the simplest case possible the turning point is supposed to be a generic turning point. This means that after translation, rescaling of the variables $(x, y)$ and rescaling of time, (3) can be written as

$$
\left\{\begin{array}{l}
\dot{x}=y \\
\dot{y}=\varepsilon\left(a(\lambda)-x+O\left(x^{2}\right)\right)-y\left(x+O\left(x^{2}\right)\right),
\end{array}\right.
$$

with $\lambda \rightarrow a(\lambda)$ a smooth submersion having 0 in its image and where the turning point is located at the origin.

We remark that traditionally, systems (4) are studied by replacing $a(\lambda)$ by an independent parameter, say $b_{0}$. Furthermore, since it is well-known that detectable canards only occur in an $O(\sqrt{\varepsilon})$-neighbourhood of $b_{0}=0$, it is customary to replace 
$\left(\varepsilon, b_{0}\right)$ by $\left(\varepsilon^{2}, \varepsilon B_{0}\right)$. Doing this, expression (3) is replaced by

$$
\left\{\begin{array}{l}
\dot{x}=y, \\
\dot{y}=\varepsilon^{2}\left(\varepsilon B_{0}-x G(x, \lambda)\right)-y f(x, \lambda),
\end{array}\right.
$$

and similarly we can replace (2) by an expression involving $\left(\varepsilon^{2}, \varepsilon B_{0}\right)$. In expression (5), we assume that $f(x, \lambda)=x+O\left(x^{2}\right)$ and that $f(x, \lambda) / x$ is strictly positive on the considered domain, in order to have a layer equation as represented in Figure 1(a). Also, we assume that $G(x, \lambda)$ is strictly positive on the considered domain, so that the "slow dynamics" $x^{\prime}=-G(x, \lambda)$ along the slow curve behaves as represented in Figure 1(b). The layer equations now contain limit periodic sets that we will call FSTS-canard cycles (fast-s slow-turning point-slow). Many papers deal with such FSTS-cycles (see e.g. [DR01]).

In such slow-fast families of vector fields (5), slow-fast cycles are parameterized by a layer variable $Y$ : for each $Y>0$, there is an orbit of the fast system through $(0, Y)$ that has a specific $\omega$-limit $(\omega(\lambda), 0)$ and $\alpha$-limit $(\alpha(\lambda), 0)$ on the slow curve $\{y=0\}$. The slow-fast cycle defined by this fast orbit, together with the slow part $[\alpha(\lambda), \omega(\lambda)] \times\{0\}$, is denoted by $\Gamma_{Y}^{\lambda}$. The condition that $G$ be positive ensures that the slow dynamics on the slow curve are regular motions from the $\omega$-limit towards the $\alpha$-limit of the fast part of $\Gamma_{Y}^{\lambda}$.

In DR01, conditions have been described on FSTS-cycles $\Gamma$ of the layer equations in (2), resp. (3), to guarantee that systems (2), resp. (3), have near $\Gamma$ (in the Hausdorff sense) a limit cycle of an a priori given multiplicity together with a full unfolding of it. The conditions are stated in terms of the slow divergence integral. A related statement in terms of a slow relation function and a fast relation function can be found in Dum, together with a slightly easier proof. The slow divergence integral, for the slow-fast cycle $\Gamma_{Y}^{\lambda}$ of Liénard equation (5), is defined as

$$
I(Y, \lambda)=\int_{\omega(\lambda)}^{\alpha(\lambda)} \frac{f(x, \lambda)^{2}}{x G(x, \lambda)} d x .
$$

For a geometrical explanation of this notion, we refer to [DMD08].

Proposition 1 ([DR01]). Consider a smooth $\left(\varepsilon, B_{0}, \lambda\right)$-family of vector fields (5), with $\varepsilon \geq 0, B_{0} \sim 0$ and $\lambda$ in a compact subset $\Lambda$ of some euclidean space. Let $f(x, \lambda) / x$ and $G(x, \lambda)$ be positive for all $\lambda$ and for all $x \in[-M, M]$. Let $Y_{0}>0$ be such that $[\alpha(\lambda), \omega(\lambda)] \subset[-M, M]$.

If the zeros of the divergence integral $I(Y, \lambda)$ undergo an elementary catastrophy of codimension $n$ at $\left(Y_{0}, \lambda_{0}\right)$, then in any Hausdorff neighbourhood of the slow-fast cycle $\Gamma_{Y}^{\lambda_{0}}$, and for $\varepsilon>0$ sufficiently small and $\lambda \sim \lambda_{0}, B_{0} \sim 0$, the family of vector fields (5) contains a limit cycle of multiplicity $n+1$ unfolded in an elementary catastrophy of codimension $n+1$.

Observe that $I(Y, \lambda)$ does not depend on $\varepsilon$, nor on $B_{0}$, and so the required condition formulated in Proposition [1 is a condition depending solely on $f(x, \lambda)$ and $G(x, \lambda)$. The presence of $B_{0}$ in (5) is however essential to obtain the required amount of limit cycles near $\Gamma_{Y}^{\lambda_{0}}$; we refer to [DR01] or [Dum] for details. We remark that a similar statement with respect to Liénard equations in the form (2) can be formulated.

Proposition 1 requires a precise checking of the necessary conditions along the FSTS-cycle in order to be applied to specific examples. Until now the proposition 
has not been applied to systems (2) or (3) in which $f(x, \lambda)$ and $g(x, \lambda)$ are polynomial in $x$, i.e. to polynomial Liénard equations. This is exactly the subject of this paper. We will essentially prove following theorems:

Theorem 1. Let $x_{0}>0$ be given. The polynomial family

$$
\left\{\begin{aligned}
\dot{x} & =y, \\
\dot{y} & =-x y+\varepsilon^{2}\left(\varepsilon B_{0}-x \varphi\left(x^{2}\right)+\sum_{i=0}^{n} a_{i} x^{2 i+2}\right),
\end{aligned}\right.
$$

where $\varphi\left(x^{2}\right)$ is an arbitrary even polynomial that is strictly positive on $\left[-x_{0}, x_{0}\right]$, and with $\varepsilon \sim 0, B_{0} \sim 0$, and $\left(a_{0}, \ldots, a_{n}\right) \sim 0$, contains a limit cycle of multiplicity $n+1$ unfolded in an elementary catastrophy of codimension $n+1$. The limit cycles are found in any a priori given small Hausdorff neighbourhood of the slow-fast cycle $\Gamma_{x_{0}}$ with $\left[-x_{0}, x_{0}\right] \times\{0\}$ as slow part.

(In fact, the function $\varphi$ need not be polynomial for the conclusions of the Theorem to be valid: any smooth, strictly positive function will do.) The relaxation oscillations obtained in Theorem 1 are of size $O(1)$, i.e. tend towards a slow-fast limit periodic set of size $O(1)$ as $\varepsilon \rightarrow 0$. We also present a result where the relaxation oscillations tend towards the origin as $\varepsilon \rightarrow 0$. This is hence a situation where the limit cycles are located in an arbitrary small neighbourhood of the turning point; nevertheless they are still relaxation oscillations.

Theorem 2. The polynomial family

$$
\left\{\begin{array}{l}
\dot{x}=y, \\
\dot{y}=-x y+\varepsilon^{2}\left(\varepsilon r^{3} B_{0}-r^{2} x+r B_{2} x^{2}-x^{3} \varphi\left(x^{2}\right)+\sum_{i=1}^{n-1} \gamma_{i} x^{2 i+2}+x^{2 n+2}\right),
\end{array}\right.
$$

where $\varphi$ is an arbitrary polynomial with $\varphi(0)>0$ and where $(\varepsilon, r) \sim(0,0), B_{0} \sim 0$, $B_{2} \sim 0$ and $\left(\gamma_{1}, \ldots, \gamma_{n-1}\right) \sim 0$, contains a limit cycle of multiplicity $n+1$ unfolded in an elementary catastrophy of codimension $n+1$. The limit cycles are found in any a priori given neighbourhood of the origin. Moreover, for a fixed and sufficiently small value of $r$ and any a priori given neighbourhood of $(r, 0)$ and any a priori given neighbourhood of $(-r, 0)$ in the phase space, the limit cycles pass through both neighbourhoods when $\varepsilon$ is sufficiently small.

Both Theorem 11 and Theorem 2 are examples of Liénard equations (3), with $\operatorname{deg}_{x} g(x, \lambda)=1$ and $\operatorname{deg}_{x} f(x, \lambda)>1$. Classical Liénard equations, on the other hand, are Liénard equations where $\operatorname{deg}_{x} f(x, \lambda)=1$ and $\operatorname{deg}_{x} g(x, \lambda) \geq 1$. The next theorem gives limit cycles of any a priori given multiplicity in a polynomial class of classical Liénard equations.

Theorem 3. Consider the polynomial family

$$
\left\{\begin{array}{l}
\dot{x}=y, \\
\dot{y}=\varepsilon^{2}\left(\varepsilon B_{0}-x\right)-y\left(x+\sum_{j=1}^{m} b_{j} x^{2 j+1}+\sum_{k=0}^{n} a_{k} x^{2 k+2}\right),
\end{array}\right.
$$

with $\varepsilon \sim 0, B_{0} \sim 0$ and $\left(a_{0}, \ldots, a_{n}\right) \sim(0, \ldots, 0)$. 
(i) If $x_{0}>0$ is given, then there exists $\delta>0$ such that for fixed $\left(b_{j}\right)_{j=1, \ldots, m}$ with $\left|b_{j}\right|<\delta, j=1, \ldots, m$, the family of vector fields (8) contains a limit cycle of multiplicity $n+1$ unfolded in an elementary catastrophy of codimension $n+1$. The limit cycles are found in any a priori given small Hausdorff neighbourhood of the slow-fast cycle $\Gamma_{x_{0}}^{b}$ (of the family (8) with $a_{k}=0$ ) with $\left[-x_{0}, x_{0}\right] \times\{0\}$ as the slow part.

(ii) For arbitrary $\left(b_{j}\right)_{j=1, \ldots, m}$ fixed, and for $x_{0}>0$ sufficiently small, the same conclusion holds as in (i).

The results that we obtain provide a reasonable amount of limit cycles for the Liénard systems under consideration. For classical Liénard equations (see Theorem 3), they do not contradict the conjecture stated in LdMP77, but provide for those systems the maximum number of limit cycles that were predicted by the conjecture. Of course that maximum had already been attained in other constructions (see [LdMP77]), however not leading to relaxation oscillations. Recall that the FSTS-canard cycles that we are working with in this paper are the simplest degenerate lps known to create a large number of limit cycles. In working with more complicated degenerate lps, as in DR07, worse can happen. Such more complicated lps have for example been used in [DPR07 to show the occurrence for some polynomial Liénard equations with more limit cycles than conjectured in LdMP77. It is also interesting to remark that, although the results in this paper agree with the [LdMP77] conjecture, some calculations in Section 4 indicate that the complexity of the proof of these results depends on the layer system that gets perturbed, hence on the precise values of $\left(b_{j}\right)_{j=1, \ldots, m}$ in Theorem 3 . For more information on the special role that singular perturbation problems play in the study of classical Liénard equations, we can refer to Rou07.

\section{Proof of Theorem 1}

Recall the family of vector fields (6):

$$
\left\{\begin{aligned}
\dot{x} & =y, \\
\dot{y} & =-x y+\varepsilon^{2}\left(\varepsilon B_{0}-x \varphi\left(x^{2}\right)+\sum_{i=0}^{n} a_{i} x^{2 i+2}\right) .
\end{aligned}\right.
$$

We observe that the linear rescaling $(x, y) \mapsto\left(x / x_{0}, y / x_{0}^{2}\right)$, together with a time rescaling and a linear rescaling of the parameters $\left(\varepsilon, B_{0}, a_{0}, \ldots, a_{n}\right)$, leaves the shape of (6) invariant and maps the slow-fast cycle $\Gamma_{x_{0}}$ to the slow-fast cycle $\Gamma_{1}$. In other words, it suffices to prove Theorem 1 for $x_{0}=1$.

To obtain a limit cycle of multiplicity $n+1$, we will use Proposition 1 from the introduction.

At $\varepsilon=0$ the divergence is given by $-x$, while the slow dynamics (i.e. the dynamics inside the center manifolds, after division by $\varepsilon^{2}$ and then taking $\varepsilon=0$ ) is given by

$$
x^{\prime}=-\varphi\left(x^{2}\right)+\sum_{i=0}^{n} a_{i} x^{2 i+1} .
$$

In Theorem 1, we require $\varphi$ to be strictly positive. Since $a \sim 0$ and $\varphi>0$, this slow dynamics is regular on $\left[-x_{0}, x_{0}\right]$ if we keep $\left|a_{i}\right|$ sufficiently small. For $\varepsilon=0$, we see that for each $x=x_{0}$ there is a fast orbit having $\left(x_{0}, 0\right)$ as the $\omega$-limit and $\left(-x_{0}, 0\right)$ as the $\alpha$-limit. We will now concentrate on the degenerate limit periodic 
set $\Gamma_{1}$ described in the formulation of Theorem 1. On the slow part of this slowfast cycle, the slow dynamics are regular (for $a_{i} \sim 0$ ) and pointing from $x=1$ to $x=-1$. As such, by Proposition 1 we will find, Hausdorff close to $\Gamma_{1}$, a limit cycle of multiplicity $n+1$ unfolded in an elementary catastrophy of codimension $n+1$ if we can prove that the slow divergence integral has at $x=1$, for well-chosen parameter values, a zero of multiplicity $n$, unfolded in an elementary catastrophy of codimension $n$. The remainder of the proof of Theorem 1 hence deals with the study of the slow divergence integral.

The slow divergence integral is given by

$$
I=I(x, a)=\int_{x}^{-x} \frac{v d v}{\varphi\left(v^{2}\right)-\sum_{i=0}^{n} a_{i} v^{2 i+1}} .
$$

We observe that in this section we can use $x>0$ as a layer variable to parameterize the slow-fast cycles instead of $Y$, as proposed in the formulation of Proposition 1. In fact the diffeomorphic relation $Y=\frac{1}{2} x^{2}$ between the two layer variables makes it clear that we may work with either variable.

For the derivative $\frac{\partial I}{\partial x}$, we have

$$
I^{\prime}:=\frac{\partial I}{\partial x}(x, a)=-\frac{2 x^{2} P(x)}{Q(x)},
$$

where

$$
\begin{aligned}
& P(x)=a_{0}+\sum_{i=1}^{n} a_{i} x^{2 i}, \\
& Q(x)=\left(\varphi\left(x^{2}\right)\right)^{2}-x^{2}(P(x))^{2} .
\end{aligned}
$$

Remark that $Q \neq 0$ when $x \sim 1$ and $(a) \sim(0)$.

If we now want the slow divergence integral $I$ to have a zero at $x=1$, then, from (9), we get the condition

$$
F(a):=\int_{1}^{-1} \frac{v d v}{\varphi\left(v^{2}\right)-\sum_{i=0}^{n} a_{i} v^{2 i+1}}=0 .
$$

The function $F$ defined here is smooth near $a=0$, and we get

$$
\frac{\partial F}{\partial a_{0}}(0,0)=\int_{1}^{-1} \frac{v^{2} d v}{\left(\varphi\left(v^{2}\right)\right)^{2}}<0 .
$$

This property, together with $F(0)=0$, shows the existence of a smooth function

$$
a_{0}=a_{0}\left(a_{1}, \ldots, a_{n}\right), \quad a_{0}(0)=0,
$$

such that $\left.F\left(a_{0}\left(a_{1}, \ldots, a_{n}\right), a_{1}, \ldots, a_{n}\right)\right)=0$, with $F$ defined in (11). For later use, we observe that

$$
\frac{\partial a_{0}}{\partial a_{i}}(0)=-\frac{\frac{\partial F}{\partial a_{i}}(0,0)}{\frac{\partial F}{\partial a_{0}}(0,0)}=-\frac{\int_{1}^{-1} \frac{v^{2 i+2} d v}{\left(\varphi\left(v^{2}\right)\right)^{2}}}{\int_{1}^{-1} \frac{v^{2} d v}{\left(\varphi\left(v^{2}\right)\right)^{2}}}
$$

and therefore, if we define

$$
A_{i}:=-\frac{\partial a_{0}}{\partial a_{i}}(0), \quad i=1, \ldots, n,
$$

we get

$$
0<A_{i}<1,
$$

and this for each $i \in\{1, \ldots, n\}$. 
From (10), we see that $I^{\prime}$ will have a zero at $x=1$ if and only if $P(1)=0$, hence if and only if

$$
a_{0}=-\sum_{i=1}^{n} a_{i}
$$

We also require that $I^{(j)}:=\frac{\partial^{j} I}{\partial x^{j}}$ be zero at $x=1$, for $2 \leq j \leq n$. While deriving the expression of $I^{(j)}(1)=0$, we will assume that $I^{\prime}(1)=I^{\prime \prime}(1)=\cdots=I^{(j-1)}(1)=0$. Since we can also assume that $I(1)=0$, we can restrict to $a_{0}=a_{0}\left(a_{1}, \ldots, a_{n}\right)$ as defined in (12). We see that

$$
I^{\prime \prime}=\frac{\partial I}{\partial x^{2}}(x, a)=-\frac{2 x^{2} P^{\prime}(x)}{Q(x)}+O(P(x))
$$

and by induction

$$
I^{(i)}=\frac{\partial^{i} I}{\partial x^{i}}(x, a)=-\frac{2 x^{2} P^{(i-1)}(x)}{Q(x)}+O\left(P(x), P^{\prime}(x), \ldots, P^{(i-2)}(x)\right),
$$

for $2 \leq i \leq n$. This implies that $I$ will have a zero of multiplicity (exactly) $n$ at $x=1$ if and only if

$$
\left\{a_{0}=a_{0}\left(a_{1}, \ldots, a_{n}\right), P(1)=P^{\prime}(1)=\cdots=P^{(n-2)}(1)=0, P^{(n-1)}(1) \neq 0\right\} .
$$

From the definition of $P$, together with (14), we see that this set of equations and inequality can be written as

$$
\left\{\begin{array}{l}
a_{0}\left(a_{1}, \ldots, a_{n}\right)+\sum_{i=1}^{n} a_{i}=0 \\
\sum_{i=1}^{n} 2 i a_{i}=0 \\
\vdots \\
\sum_{i=1}^{n} 2 i(2 i-1) \ldots(2 i-j) a_{i}=0 \\
\vdots \\
\sum_{i=1}^{n} 2 i(2 i-1) \ldots(2 i+3-n) a_{i}=0,
\end{array}\right.
$$

together with the inequality

$$
\sum_{i=1}^{n} 2 i(2 i-1) \ldots(2 i+2-n) a_{i} \neq 0
$$

when (15) holds. Each line in the left-hand side of (15) or (16) can be expanded in powers of $i$, inducing that

$$
\sum_{i=1}^{n} 2 i(2 i-1) \ldots(2 i-j) a_{i}=\sum_{i=1}^{n}\left(\sum_{k=1}^{j+1} c_{k} i^{j}\right) a_{i}
$$

for some integers $c_{k}$ not depending on $i$. It now follows that conditions (15), together with (16), can be rewritten as

$$
\left\{\begin{array}{l}
F_{1}(a):=a_{0}\left(a_{1}, \ldots, a_{n}\right)+\sum_{i=1}^{n} a_{i}=0 \\
F_{\ell+1}(a):=\sum_{i=1}^{n} i^{\ell} a_{i}=0, \quad \ell=1, \ldots, n-2
\end{array}\right.
$$

(if we use $a$ as a shortcut for $\left(a_{1}, \ldots, a_{n}\right)$ ), with $\sum_{i=1}^{n} i^{n-1} a_{i} \neq 0$ when (17) holds. Now, based on the Implicit Function Theorem, (17) will have a smooth 
solution $\left(a_{1}\left(a_{n}\right), \ldots, a_{n-1}\left(a_{n}\right)\right)$ near $a_{n}=0$, with $\left(a_{1}(0), \ldots, a_{n-1}(0)\right)=0$ if $\frac{\partial\left(F_{1}, \ldots, F_{n-1}\right)}{\partial\left(a_{1}, \ldots, a_{n-1}\right)}(0) \neq 0$, hence if

$$
\left|\begin{array}{cccc}
1-A_{1} & 1-A_{2} & \cdots & 1-A_{m} \\
1 & 2 & & m \\
1 & 2^{2} & \ddots & m^{2} \\
\vdots & \vdots & & \vdots \\
1 & 2^{m-1} & \cdots & m^{m-1}
\end{array}\right| \neq 0
$$

where $m=n-1$ and where the $A_{i}$ are defined in (13). We will now prove (18) for any value of $m$, including the requested $m=n-1$, but also $m=n$. In the latter case, this implies that when (17) holds, then necessarily the inequality $F_{n-1}(a) \neq 0$ has to hold for $a=\left(a_{1}, \ldots, a_{n}\right) \neq 0$ as well.

To prove (18) for any value of $m$ we use the precise value of $A_{i}$ as defined in (13), in the form of a quotient of two rational integrals. We clearly see that (18) is true if we can prove that

$$
\left|\begin{array}{cccc}
\int_{1}^{-1} \frac{v^{2}\left(1-v^{2}\right) d v}{\left(\varphi\left(v^{2}\right)\right)^{2}} & \int_{1}^{-1} \frac{v^{2}\left(1-v^{4}\right) d v}{\left(\varphi\left(v^{2}\right)\right)^{2}} & \cdots & \int_{1}^{-1} \frac{v^{2}\left(1-v^{2 m}\right) d v}{\left(\varphi\left(v^{2}\right)\right)^{2}} \\
1 & 2 & & m \\
1 & 2^{2} & \ddots & m^{2} \\
\vdots & \vdots & & \vdots \\
1 & 2^{m-1} & \cdots & m^{m-1}
\end{array}\right| \neq 0,
$$

for any value of $m$. The left-hand side of (19) is exactly $\int_{1}^{-1} \frac{v^{2}}{\left(\varphi\left(v^{2}\right)\right)^{2}} D_{m}\left(v^{2}\right) d v$, where $D_{m}$ is given by

$$
D_{m}(w):=\left|\begin{array}{cccc}
1-w & 1-w^{2} & \cdots & 1-w^{m} \\
1 & 2 & & m \\
1 & 2^{2} & \ddots & m^{2} \\
\vdots & \vdots & & \vdots \\
1 & 2^{m-1} & \cdots & m^{m-1}
\end{array}\right| .
$$

So for sure, (18) is true if we prove that $D_{m}(w)$ stays nonzero for $w \in[0,1[$. Now, $D_{m}$ defines a polynomial in $w$ of degree $m$ that has a zero at $w=1$. In considering $\frac{\partial^{i} D_{m}}{\partial w^{i}}$, with $i=1, \ldots, m-1$, we get a linear relation between the first $i+1$ rows for $w=1$. It implies that $D_{m}(1)=D_{m}^{\prime}(1)=\cdots=D_{m}^{(m-1)}(1)=0$, so that

$$
D_{m}(w)=C_{m}(1-w)^{m}, \quad C_{m}:=\left|\begin{array}{cccc}
1 & 2 & \cdots & m-1 \\
\vdots & \vdots & & \vdots \\
1 & 2^{m-1} & \cdots & (m-1)^{m-1}
\end{array}\right| .
$$

The constant $C_{m}$ is nonzero, as it can be expressed as $m$ ! multiplied by a Vandermonde determinant. This proves our claim.

The curve in parameter space, along which the slow divergence integral $I$ has, at $x=1$, a zero of multiplicity (exactly) equal to $n$, is given by the smooth function

$$
\begin{aligned}
& a_{i}=a_{i}\left(a_{n}\right), \quad i=1, \ldots, n-1, \quad \text { with } a_{i}(0)=0, \\
& a_{0}=a_{0}\left(a_{1}, \ldots, a_{n}\right) .
\end{aligned}
$$


We still need to show that the zero of multiplicity $n$ gets unfolded in an elementary catastrophy of codimension $n$.

From [GG73, we know that it suffices to prove that

$$
\operatorname{det}\left(\frac{\partial\left(I, I^{\prime}, \ldots, I^{(n-2)}\right)}{\partial\left(a_{1}, \ldots, a_{n-1}\right)}\right) \neq 0
$$

for $x=1$ and $a_{0}=a_{0}\left(a_{1}, \ldots, a_{n}\right)$. This property will clearly follow if we can prove that it is nonzero at $x=1$ and $a=0$.

From (9) and (10), we get

$$
\frac{\partial I}{\partial a_{i}}(1,0,0)=\int_{-1}^{1} \frac{v^{2 i+2} d v}{\left(\varphi\left(v^{2}\right)\right)^{2}}, \quad \frac{\partial I^{\prime}}{\partial a_{i}}(1,0,0)=-\frac{1}{2} .
$$

It is also easy to check that

$$
\frac{\partial I^{(j)}}{\partial a_{i}}(1,0,0)=-\frac{1}{4}(2 i(2 i-1) \ldots(2 i+2-j)), \quad \text { for } j=2, \ldots, n-2 .
$$

As in the study of (19) it is clear that these values will imply that expression (20) will surely be nonzero when the following determinant is nonzero for all $w \in[0,1]$ :

$$
E_{n-1}(w):=\left|\begin{array}{cccc}
w & w^{2} & \ldots & w^{n-1} \\
1 & 1 & & 1 \\
1 & 2 & \ddots & n-1 \\
\vdots & \vdots & & \vdots \\
1 & 2^{n-3} & \cdots & (n-1)^{n-3}
\end{array}\right| .
$$

By checking the multiplicity of the zero that the polynomial $E_{n-1}$ has at $w=1$, we again find that

$$
E_{n-1}(w)=E_{n-1}^{\prime}(0) w(1-w)^{n-2},
$$

with

$$
E_{n-1}^{\prime}(0)=\left|\begin{array}{cccc}
1 & 1 & \cdots & 1 \\
2 & 3 & \cdots & n-1 \\
\vdots & \vdots & & \vdots \\
2^{n-3} & 3^{n-3} & \cdots & (n-1)^{n-3}
\end{array}\right| .
$$

This expression is a well-known Vandermonde determinant, which is nonzero. This proves the claim and finishes the proof of Theorem 1 .

\section{Proof of Theorem 2}

We consider the polynomial family

$$
\left\{\begin{array}{l}
\dot{x}=y, \\
\dot{y}=-x y+\varepsilon^{2}\left(\varepsilon r^{3} B_{0}-r^{2} x+r B_{2} x^{2}-x^{3} \varphi\left(x^{2}\right)+\sum_{i=1}^{n} \gamma_{i} x^{2 i+2}\right),
\end{array}\right.
$$

with $(\varepsilon, r) \sim(0,0), \varepsilon \geq 0, B_{2} \sim 0, B_{0} \sim 0, \gamma=\left(\gamma_{1}, \ldots, \gamma_{n}\right)$ with $\gamma_{n}=1$ and $\left(\gamma_{1}, \ldots, \gamma_{n-1}\right) \sim 0$. We make a quasi-homogeneous blow up at $(x, y, r)=(0,0,0)$. In this paper, it suffices to study the traditional rescaling chart (the family chart): we write

$$
(x, y)=\left(r \bar{x}, r^{2} \bar{y}\right) .
$$


It changes (21), after division by $r$, into

$$
\left\{\begin{array}{l}
\dot{\bar{x}}=\bar{y} \\
\dot{\bar{y}}=-\bar{x} \bar{y}+\varepsilon^{2}\left(\varepsilon B_{0}-\bar{x} \bar{\varphi}\left(\bar{x}^{2}, r^{2}\right)+B_{2} \bar{x}^{2}+\sum_{i=1}^{n} \gamma_{i} r^{2 i-1} \bar{x}^{2 i+2}\right),
\end{array}\right.
$$

where $\bar{\varphi}\left(x^{2}, r^{2}\right)=1+\bar{x}^{2} \varphi\left(r^{2} \bar{x}^{2}\right)$ is strictly positive for $r$ sufficiently small. Let $\Gamma_{\bar{x}_{0}}$ be the slow-fast cycle corresponding to $\bar{x}_{0}$, i.e. the slow-fast cycle with slow part $\left[-\bar{x}_{0}, \bar{x}_{0}\right] \times\{0\}$ and with fast part $\left\{\bar{y}=\frac{1}{2} \bar{x}_{0}^{2}-\frac{1}{2} \bar{x}^{2}, \bar{y} \geq 0\right\}$. The slow divergence integral for this slow-fast cycle is given by

$$
I\left(\bar{x}_{0}, B_{2}, \gamma, r\right)=\int_{\bar{x}_{0}}^{-\bar{x}_{0}} \frac{v d v}{\bar{\varphi}\left(v^{2}, r^{2}\right)-B_{2} v-\sum_{i=1}^{n} \gamma_{i} r^{2 i-1} v^{2 i+1}} .
$$

Let us introduce

$$
a_{0}=B_{2}, \quad a_{i}=\gamma_{i} r^{2 i-1}, \quad i=1, \ldots, n .
$$

In terms of the rescaled coordinates $(\bar{x}, \bar{y})$, we get a singular perturbation problem that satisfies the hypotheses of Theorem 1, Following the proof of this theorem and defining

$$
\bar{I}\left(\bar{x}_{0}, a, r\right)=I\left(\bar{x}_{0}, a_{0},\left(a_{i} / r^{2 i-1}\right), r\right),
$$

we know that $\bar{I}$ undergoes, at $\bar{x}_{0}=1$, an elementary catastrophy of codimension $n$. The curve in parameter space, along which the slow divergence integral $\bar{I}$ has, at $\bar{x}=1$, a zero of multiplicity (exactly) equal to $n$, is given by the smooth function

$$
\begin{aligned}
a_{i} & =a_{i}\left(a_{n}\right), \quad i=1, \ldots, n-1, \quad \text { with } a_{i}(0)=0, \\
a_{0} & =a_{0}\left(a_{1}, \ldots, a_{n}\right) .
\end{aligned}
$$

From (23) and from the fact that $\gamma_{n}=1$ and hence $a_{n}=r^{2 n-1}$, it is clear that for $r \sim 0$ we can parameterize this curve in $\left(\gamma, B_{2}\right)$-space by $r$. The curve can be represented by smooth functions

$$
\begin{aligned}
\gamma_{i} & =\gamma_{i}(r), \quad i=1, \ldots, n-1, \quad \text { with } \gamma_{i}(0)=0, \\
B_{2} & =B_{2}(r) \quad \text { with } B_{2}(0)=0 .
\end{aligned}
$$

To show that for each value $r>0, r \sim 0$, the zero of multiplicity $n$ gets unfolded in an elementary catastrophy of codimension $n$, we know from GG73] that it suffices to prove that

$$
\operatorname{det}\left(\frac{\partial\left(I, I^{\prime}, \ldots, I^{(n-2)}\right)}{\partial\left(\gamma_{1}, \ldots, \gamma_{n-1}\right)}\right) \neq 0,
$$

for $\bar{x}=1, r>0$ and $\left(B_{2}, \gamma\right)=\left(B_{2}(u), \gamma(u)\right)$. This property clearly follows from the fact that

$$
\operatorname{det}\left(\frac{\partial\left(\bar{I}, \bar{I}^{\prime}, \ldots, \bar{I}^{(n-2)}\right)}{\partial\left(a_{1}, \ldots, a_{n-1}\right)}\right)
$$

is nonzero at $(\bar{x}, r)=(1,0)$, i.e. at $\bar{x}=1,\left(a_{0}, a_{1}, \ldots, a_{n}\right)=0$, which has been proven during the proof of Theorem 1 


\section{Proof of Theorem 3}

Consider

$$
\left\{\begin{array}{l}
\dot{x}=y \\
\dot{y}=\varepsilon^{2}\left(\varepsilon B_{0}-x\right)-y\left(f(x, b)+x^{2} h(x, a)\right)
\end{array}\right.
$$

with $f(x, b)=x+\sum_{j=1}^{m} b_{j} x^{2 j+1}$ and $h(x, a)=\sum_{k=0}^{n} a_{k} x^{2 k}$. Wherever we consider $f(x, b)$, we will suppose that $f(x) / x$ is strictly positive. As before, we intend to apply Proposition 1 by checking properties of the slow divergence integral of slowfast cycles.

An extra complication arises in the context of classical Liénard equations when compared to the contexts of Theorem 1 or Theorem 2 in the setting of this section, the fast relation function, as defined in the layer equation at $\varepsilon=0$, changes under influence of the perturbation parameter $a$ (recall that $b$ is fixed). We will hence not be able to give an explicit expression of the slow divergence integral, but we will work with the linearization of the slow divergence integral about $a=0$, as will become clear in the proof below.

In order to better deal with the changing fast relation function, we prefer to change (24) by the equivalent system

$$
\left\{\begin{array}{l}
\dot{x}=y-F(x)-x^{3} H(x, a) \\
\dot{y}=\varepsilon^{2}\left(\varepsilon B_{0}-x\right)
\end{array}\right.
$$

where $F(x)=\int_{0}^{x} f(s, b) d s$ (dropping the dependence on $b$ in the notation of $F$ and recalling that we keep $b$ fixed), and

$$
H(x, a):=x^{-3} \int_{0}^{x} s^{2} h(s, a) d s=\sum_{k=0}^{n} a_{k} \frac{x^{2 k}}{2 k+3} .
$$

Given $x_{0}>0$, we define the slow-fast cycle $\Gamma_{x_{0}}^{a}$ as the cycle composed of the fast part $\left\{y=y_{0}, F(x)+x^{3} H(x, a) \leq y_{0}\right\}$ and the slow part $\left\{y=F(x)+x^{3} H(x, a), y \leq\right.$ $\left.y_{0}\right\}$, where $y_{0}=F\left(x_{0}\right)+x_{0}^{3} H\left(x_{0}, a\right)$. Of course, $\Gamma_{x_{0}}^{0}=\Gamma_{x_{0}}$. The fast part is a horizontal segment, ending to the right at $\left(x_{0}, y_{0}\right)$ and starting to the left at $\left(x_{1}\left(x_{0}, a\right), y_{0}\right)$, where

$$
F\left(x_{1}\right)+x_{1}^{3} H\left(x_{1}, a\right)=F\left(x_{0}\right)+x_{0}^{3} H\left(x_{0}, a\right), \quad x_{1}\left(x_{0}, 0\right)=-x_{0} .
$$

Using implicit differentiation and employing the fact that $F$ is even, one easily finds

$$
\frac{\partial x_{1}}{\partial a_{i}}(x, 0)=-\frac{2 x^{3+2 i}}{\left((2 k+3) F^{\prime}(x)\right.} .
$$

We now define the slow divergence integral

$$
I(x, a)=\int_{x_{1}(x, a)}^{x} \frac{\left(\frac{\partial}{\partial x}\left(F(x)+x^{3} H(x, a)\right)\right)^{2}}{x} d x .
$$

We remark that $I(x, 0)=\int_{-x}^{x} \frac{F^{\prime}(x)^{2}}{x} d x=0$, again using the fact that $F$ is even. We write

$$
I(x, a)=\sum_{i=0}^{n} I_{i}(x) a_{i}+O\left(\|a\|^{2}\right) .
$$


We find

$$
\begin{aligned}
I_{i}(x) & =\frac{F^{\prime}(x)^{2}}{x}\left(\frac{-2 x^{3+2 i}}{(3+2 i) F^{\prime}(x)}\right)+\int_{-x}^{x} \frac{2 F^{\prime}(v)(3+2 i) v^{2+2 i}}{v} d v \\
& =-\frac{2}{3+2 i} f(x) x^{2+2 i}+2 \int_{-x}^{x} f(v) v^{1+2 i} d x .
\end{aligned}
$$

Let us now calculate these integrals in detail using $f(x)=\sum_{j=0}^{m} b_{j} x^{2 j+1}$ (with the convention $\left.b_{0}:=1\right)$ :

$$
\begin{aligned}
I_{i}(x) & =\sum_{j=0}^{m} b_{j}\left(-\frac{2}{3+2 i} x^{2 j+1} x^{2+2 i}+2 \int_{-x}^{x} v^{2 j+1} v^{1+2 i} d x\right) \\
& =\sum_{j=0}^{m} b_{j}\left(-\frac{2}{3+2 i}+\frac{4}{2 i+2 j+3}\right) x^{2 j+2 i+3} \\
& =\frac{2}{2 i+3} x^{2 i+3}\left(1+\sum_{j=1}^{m} b_{j} \frac{2 i-2 j+3}{2 i+2 j+3} x^{2 j}\right) .
\end{aligned}
$$

We are now in a position to prove Theorem 3 . First let $x_{0}>0$ be arbitrary, and let $\left|b_{j}\right|<\delta$, where $\delta$ is yet to be specified. This is the setting of part (i) of the theorem. If we let $b=\left(b_{1}, \ldots, b_{m}\right)$, then $\|b\|=O(\delta)$ and

$$
I_{i}(x)=\frac{2}{2 i+3} x^{2 i+3}(1+O(\delta)),
$$

if we keep $x$ in some interval $[-M, M]$ containing $x_{0}$. Similarly, for arbitrary $b$, we have the same property (26), provided we keep $x_{0}>0$ in an $O(\delta)$-neighbourhood of the origin. This is the setting of part (ii) of the theorem. In the remainder of the proof we only use property (26), this way giving the same proof for both parts of the theorem.

For $\delta$ small enough, $I_{i}\left(x_{0}\right)>0$, so by the Implicit Function Theorem we find

$$
a_{0}=a_{0}\left(a_{1}, \ldots, a_{n}\right) \text { such that } I\left(x_{0}, a_{0}\left(a_{1}, \ldots, a_{n}\right), a_{1}, \ldots, a_{n}\right)=0 .
$$

Even better, it is not hard to see that

$$
\frac{\partial\left(I, I^{\prime}, \ldots, I^{(n-1)}\right)}{\partial\left(a_{0}, \ldots, a_{n-1}\right)}=C+O(\delta)
$$

for some nonzero constant $C$. This shows, for $\delta$ small enough, the existence of a curve

$$
a_{0}=a_{0}\left(a_{n}\right), a_{1}=a_{1}\left(a_{n}\right), \ldots, a_{n-1}=a_{n-1}\left(a_{n}\right)
$$

in parameter space along which $I(x, a)$ has a zero of multiplicity $n$ at $x_{0}$. Of course, this curve lies inside the manifold $a_{0}=a_{0}\left(a_{1}, \ldots, a_{n}\right)$. The observation that

$$
\left.\frac{\partial\left(I, I^{\prime}, \ldots, I^{(n)}\right)}{\partial\left(a_{0}, \ldots, a_{n}\right)}\right|_{x=x_{0}, a=0}=C^{\prime}+O(\delta)
$$

for some $C^{\prime} \neq 0$ should suffice to see that the multiplicity of the zero is not greater than $n$ (again taking $\delta$ small enough). Finally, we prove that $I$ undergoes an 
elementary catastrophy of codimension $n$, using the condition found in GG73]: it suffices to prove

$$
\frac{\partial\left(I, I^{\prime}, \ldots, I^{(n-2)}\right)}{\partial\left(a_{1}, \ldots, a_{n-1}\right)} \neq 0
$$

along the curve $\left\{a_{i}=a_{i}\left(a_{n}\right)\right\}_{i=0, \ldots, n}$. Again, for $\delta$ small enough, this is easy to see. This proves Theorem 3 .

Remark. In view of Proposition 1 one might believe that the condition on the $\left(b_{j}\right)$ would be that $\frac{f(x)}{x}=1+\sum_{j=1}^{m}(2 j+2) b_{j} x^{2 j}$ is strictly positive on $\left[-x_{0}, x_{0}\right]$. This condition expresses that the origin is the only turning point of the slow curve on the interval $x \in\left[-x_{0}, x_{0}\right]$. It reveals not to be a sufficient condition to prove Theorem 3 using the method that has been employed above, explaining the further restrictions imposed in the statement.

\section{REFERENCES}

[DMD08] P. De Maesschalck and F. Dumortier, Canard cycles in the presence of slow dynamics with singularities, Proc. Roy. Soc. Edinburgh Sect. A 138 (2008), no. 2, 265-299. MR 2406691

[DPR07] Freddy Dumortier, Daniel Panazzolo, and Robert Roussarie, More limit cycles than expected in Liénard equations, Proc. Amer. Math. Soc. 135 (2007), no. 6, 1895-1904 (electronic). MR2286102 (2007m:34081)

[DR01] F. Dumortier and R. Roussarie, Multiple canard cycles in generalized Liénard equations, J. Differential Equations 174 (2001), no. 1, 1-29. MR.1844521 (2002k:34076)

[DR07] Freddy Dumortier and Robert Roussarie, Bifurcation of relaxation oscillations in dimension two, Discrete Contin. Dyn. Syst. 19 (2007), no. 4, 631-674. MR2342266 (2008k:34164)

[Dum] F. Dumortier, Slow divergence integral and balanced canard solutions, Qualitative Theory of Dynamical Systems, to appear.

[GG73] M. Golubitsky and V. Guillemin, Stable mappings and their singularities, Graduate Texts in Mathematics, Vol. 14, Springer-Verlag, New York, 1973. MR0341518(49:6269)

[LdMP77] A. Lins, W. de Melo, and C. C. Pugh, On Liénard's equation, Geometry and topology (Proc. III Latin Amer. School of Math., Inst. Mat. Pura Aplicada CNPq, Rio de Janeiro, 1976), Lecture Notes in Math., Vol. 597, Springer, Berlin, 1977, pp. 335-357. MR0448423(56:6730)

[Rou07] R. Roussarie, Putting a boundary to the space of Liénard equations, Discrete Contin. Dyn. Syst. 17 (2007), no. 2, 441-448. MR2257444 (2007i:34046)

Hasselt University, Campus Diepenbeek, Agoralaan gebouw D, B-3590 Diepenbeek, BELGIUM

E-mail address: peter.demaesschalck@uhasselt.be

Hasselt University, Campus Diepenbeek, Agoralaan gebouw D, B-3590 Diepenbeek, BELGIUM

E-mail address: freddy.dumortier@uhasselt.be 